\title{
Alteration of Porcine Serum Albumin Levels in Pork Meat by Marination in Kiwi or Pineapple Juice and Subsequent Pan Broiling
}

\author{
Sung-Sil Moon ${ }^{1, \dagger}$, Dongwook Kim ${ }^{\dagger}$, II-Suk Kim², Jun-Sang Ham³ ${ }^{3}$ Beom-Young Park², and Aera Jang* \\ Department of Animal Products and Food Science, Kangwon National University, Chuncheon 200-701 Korea \\ ${ }^{1}$ Sunjin Meat Research Center, Seoul 134-060, Korea \\ ${ }^{2}$ Department of Animal Resources Technology, Gyeongnam National University of Science and Technology, \\ Gyeongnam 660-758, Korea \\ ${ }^{3}$ National Institute of Animal Science, RDA, Suwon 441-706, Korea
}

\begin{abstract}
This study was conducted to evaluate the changes in porcine serum albumin (PSA), a major allergen, which occur when raw pork ham is marinated with kiwi or pineapple juice, and/or when the ham is pan broiled at $300^{\circ} \mathrm{C}$ for 4 min after marination. In this study, raw pork ham was soaked for $4 \mathrm{~h}$ or $8 \mathrm{~h}$ in marinades containing commercial marinating sauce only, commercial marinating sauce and $7 \%$ kiwi juice, or commercial marinating sauce and $7 \%$ pineapple juice. When the meat was marinated and then pan-broiled, pork ham meat protein was significantly denatured and hydrolyzed, and the level of PSA in the meat was significantly reduced. The PSA contents of pork broiled without marination, pork that had been marinated in commercial marinating sauce alone, pork that had been marinated in commercial marinating sauce with kiwi juice, and pork that had been marinated in commercial marinating sauce with pineapple juice, were 95.4, 43.3, 14.3, and 5.4 ng/ $\mathrm{mL}$, respectively $(p<0.05)$. Marinating with pineapple juice was more effective than marinating with kiwi juice; and marination for $8 \mathrm{~h}$ was more effective than marinating for $4 \mathrm{~h}$. These results indicate that the level of PSA in pork ham is effectively reduced, when the meat is first marinated in sauces that contain kiwi or pineapple extracts for $8 \mathrm{~h}$, rather than $4 \mathrm{~h}$, and then cooked. Further study is needed to determine whether marinated pork meat reduces allergenicity in vivo, as well.
\end{abstract}

Key words: porcine serum albumin, marinating, allergen, sandwich ELISA, pork ham

\section{Introduction}

The term food allergy is used to describe adverse immune responses to foods (Sicherer and Sampson, 2010). Food allergy rates vary by age, local diet, and various other factors. Food-induced allergic reactions are responsible for a variety of symptoms and disorders involving the skin and gastrointestinal and respiratory tracts and can be attributed to IgE-mediated, non-IgE-mediated, and mixed-type mechanisms. Recently, scientists have reported IgG antibody in food allergy patients and $\mathrm{Fc}$ receptor of IgG4 antibody on the surface of mast cell (Chen et al., 2008; Lee et al., 1993). Since then scientists also focus on IgG4 antibody to explain food allergy which is non-IgE

\footnotetext{
*Corresponding author: Aera Jang, Department of Aniaml Products and Food Science, Kangwon National University, Chuncheon 200-701, Korea. Tel: +82-33-250-8643, Fax: +82-33259-5574, E-mail: ajang@kangwon.ac.kr

${ }^{\dagger}$ Both authors contributed equally to this work.
}

mediated (Cho et al., 2001; Lee et al., 1993). Food allergens have a number of common characteristics: they are water-soluble glycoproteins, 10 to $70 \mathrm{kDa}$ in size, and relatively resistant to acid, heat, and proteases (Sicherer and Sampson, 2010).

Pork is the most popular meat in Korea, and the level of pork meat consumption has increased in recent years. Pork meat consumption per capita in 2005 was $17.8 \mathrm{~kg}$ and rose to $19.2 \mathrm{~kg}$ in 2012 (Korea Meat Trade Association, 2014). However, a survey of urban preschool children revealed that pork was one of the ten foods most often associated with allergy, as the incidence of pork allergy among the children was 1.9\% (Chung et al., 2001). Although the incidence seems low, the consequence is severe and results in anaphylaxis shock. Moreover, pork has been classified as a major food allergen (Kim et al., 2011) and, as per Article 10 of the Korea Food Sanitation Act, must be indicated as such on labels for food products.

The most well-known allergens from meat are serum albumin $(66 \mathrm{kDa})$, gamma-globulin $(60 \mathrm{kDa})$, and actin 
(42 $\mathrm{kDa}$ ), as well as several additional proteins of various sizes $(14,18,20,45$, and more than $60 \mathrm{kDa})$. These allergens are found in not only beef but also pork and lamb and can cause cross-reactivity among foods (Fiocchi et al., 2000). Purified porcine serum albumin (PSA) or extracts from unprocessed meats that contain PSA have been used not only to evaluate allergenic potential but also to study the feasibility of reducing allergenicity by means of heat processing (Kim et al., 2011).

Current management of food allergies consists of educating the patient to avoid ingesting the responsible allergen and to initiate therapy (e.g., by injecting epinephrine to treat anaphylaxis) in case of unintended ingestion (Sicherer and Sampson, 2010). However, heating is one of the most efficient methods to reduce allergenicity. Fisher (1982) reported the case of a patient who presented with anaphylactic symptoms after ingesting rare-cooked beef but who was able to tolerate well-cooked beef. Although treatment of meat with digestive enzymes has been reported to reduce the allergenicity of serum albumin from different species (Kim et al., 2011), there has been no specific study examining the effects of fruit enzymes and pan broiling at $300^{\circ} \mathrm{C}$ on altering the allergen PSA. Therefore, the aim of this study was to use SDS-PAGE, immunoblotting, and sandwich ELISA to determine the effect of pan broiling and/or marinating with kiwi or pineapple juice on the level of PSA in pork meat.

\section{Materials and Methods}

\section{Marination and pan broiling}

Raw pork ham, commercial marinating sauce, kiwis, and pineapples were purchased from a local market. Raw pork ham was soaked for $4 \mathrm{~h}$ or $8 \mathrm{~h}$ in a marinade containing $27 \%$ commercial sauce only, commercial sauce with $10 \%$ kiwi juice, or a commercial sauce with $10 \%$ pineapple juice (Table 1). Then, it was either analyzed immediately or subjected to pan broiling on a hot plate for $4 \mathrm{~min}$ at $300^{\circ} \mathrm{C}$ prior to analysis (Fig. 1). In addition, pork that was pan broiled without prior marination was also examined.

\section{Porcine serum albumin (PSA) extraction}

PSA was extracted from the pork using the method described in Wang et al. (2002), with a slight modification. Twenty grams of meat was added to $40 \mathrm{~mL}$ of 0.01 $\mathrm{M}$ phosphate-buffered saline (PBS, pH 7.3). Next, the meat was homogenized at $10,000 \mathrm{rpm}$ for $30-60 \mathrm{sec}$ and then centrifuged at $16,000 \mathrm{~g}$ for $30 \mathrm{~min}$. The supernatant was filtered using Whatman No. 1 filter paper and then used for further experiments.

\section{Commercial PSA and antibodies}

Commercial PSA and a horseradish-peroxidase (HRP)conjugated rabbit anti-goat-IgG antibody were purchased from Sigma Chemical Co. (USA). A goat anti-PSA IgG antibody was obtained from Bethyl Laboratories Inc. (USA).

\section{SDS-PAGE}

Proteins from pork meat extracts were separated according to their molecular weights on $12 \%$ separating gels and 5\% stacking gels. Prior to loading, the samples were analyzed for protein content using a BCA protein assay kit (B9643, Sigma). Coomassie Brilliant Blue R 250 (Sigma) was used for visualization (Laemmli, 1970). Electrophoresis was performed using a miniPAGE (page Run, AE-6531; ATTO, Japan) system at $100 \mathrm{~V}$ for $3 \mathrm{~h}$. The sizes of the protein bands were identified using a standard Prosi prestained protein marker (GenDEPOT, USA).

\section{Immunoblotting assay}

After SDS-PAGE, proteins were transferred to methanol-activated polyvinylidene difluoride (PVDF) membranes and then to immobilization paper (Shleicher \& Schuell, USA) for $30 \mathrm{~min}$ at $200 \mathrm{~mA}$ using an electrophoresis power supply (Northeastern Science Company, USA) and a Mini Trans-Blot Transfer Cell (Bio-Rad Laboratories). The PVDF membranes were blocked with $3 \%$ gelatin in TBS-T (20 mM Tris, $137 \mathrm{mM} \mathrm{NaCl}$, pH 7.6, with $0.1 \%$

Table 1. Manufacturing compositions of pork meat marinated with commercial sauce alone or with kiwi or pineapple juice

\begin{tabular}{ccccc}
\hline \hline & CON & RPM & RPMK & RPMP \\
\hline Raw pork ham & 100 & 100 & 100 & 100 \\
Commercial marinating sauce & - & 27 & 27 & 10 \\
Kiwi juice & - & - & - & - \\
Pineapple juice & - & 127 & 137 & 10 \\
\hline Total & 100 & -137
\end{tabular}

CON, without marinating; RPM, raw pork ham marinated with commercial sauce; RPMK, raw pork ham marinated with commercial sauce and kiwi juice; RPMP, raw pork ham marinated with commercial sauce and pineapple juice. 
Tween-20) for $2 \mathrm{~h}$ at RT and then washed in TBS-T 3 times for 10 min each time. The membranes were then incubated with the primary antibody (diluted 1:1000 in TBS-T) overnight with rocking at $4{ }^{\circ} \mathrm{C}$. Then the membrane was washed again in TBS-T 3 times for 10 min each time. Then, an HRP-labeled rabbit anti-goat IgG antibody (diluted 1:3000 in TBS-T) was added to the membranes, which were then incubated for $1 \mathrm{~h}$ at RT and subsequently washed 3 times in TBS-T. Antigen-antibody complexes were detected by chemiluminescence (Santa Cruz Biotechnologies). Chemiluminescence signals were observed using a ChemiDoc (Vilver Lourmat, France).

\section{Sandwich ELISA assay}

For the sandwich assay, 96-well flat-bottomed microplates were coated overnight at $4^{\circ} \mathrm{C}$ with $100 \mu \mathrm{L} /$ well of $0.05 \mathrm{M}$ carbonate-bicarbonate buffer ( $\mathrm{pH}$ 9.6) containing an affinity-purified anti-PSA antibody $(1 \mu \mathrm{L}$ of the affinity-purified antibody was diluted in $100 \mu \mathrm{L}$ coating buffer for each well to be coated). The plates were then washed 5 times in TBS containing $0.05 \%$ Tween 20 (pH 7.6). After washing, the wells were blocked with $200 \mu \mathrm{L} /$ well of a blocking solution consisting of $1 \%$ gelatin in TBS and then incubated for $1 \mathrm{~h}$ at RT. After washing again, $100 \mu \mathrm{L} /$ well of a standard at concentrations ranging from 7.8 to $500 \mathrm{ng} / \mathrm{mL}$. Standards, buffer blanks (assay buffer), and sample extracts were incubated for $1 \mathrm{~h}$ at RT under gentle shaking in triplicate on each plate. After washing, the plates were incubated with $100 \mu \mathrm{L} /$ well of HRPdetection antibody (diluted 1:100,000) for $1 \mathrm{~h}$ at RT. All dilutions were performed in assay buffer, and the plates were sealed with plate-sealing film during incubations. After a final wash, each well was incubated with $100 \mu \mathrm{L} /$ well TMB substrate solution. The plate was developed in the dark at room temperature for $15 \mathrm{~min}$. Color development was stopped by the addition of $100 \mu \mathrm{L} /$ well of ELISA stop solution $\left(0.18 \mathrm{M} \mathrm{H}_{2} \mathrm{SO}_{4}\right)$. The plate was evaluated within $30 \mathrm{~min}$ of stopping the reaction by measuring the absorbance at $450 \mathrm{~nm}$ using a Spectra Max $\mathrm{M}^{2 \mathrm{e}}$ Microplate Reader (Molecular Devices, USA).

\section{Statistical analysis}

Data were analyzed using the general linear model, and significant differences were determined with Duncan's multiple range test at $p<0.05$ using SAS software (SAS Institute Inc., USA).

\section{Results and Discussion}

\section{Marinating and broiling pork ham}

Fig. 1 shows a diagram of experimental treatments and the sample preparation procedure used in this study. Raw

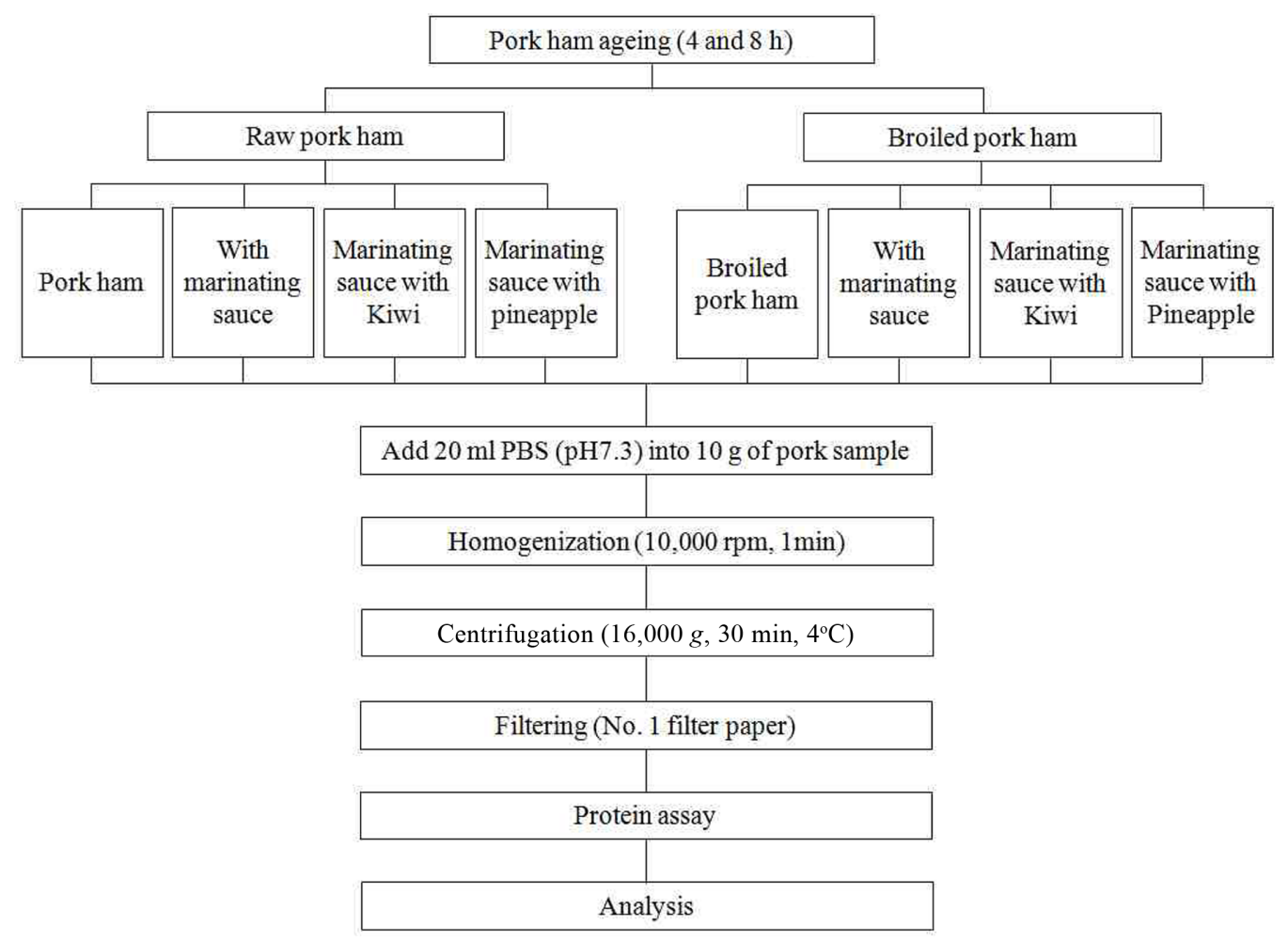

Fig. 1. Diagram showing experimental treatments and sample preparation procedure. 
(A)

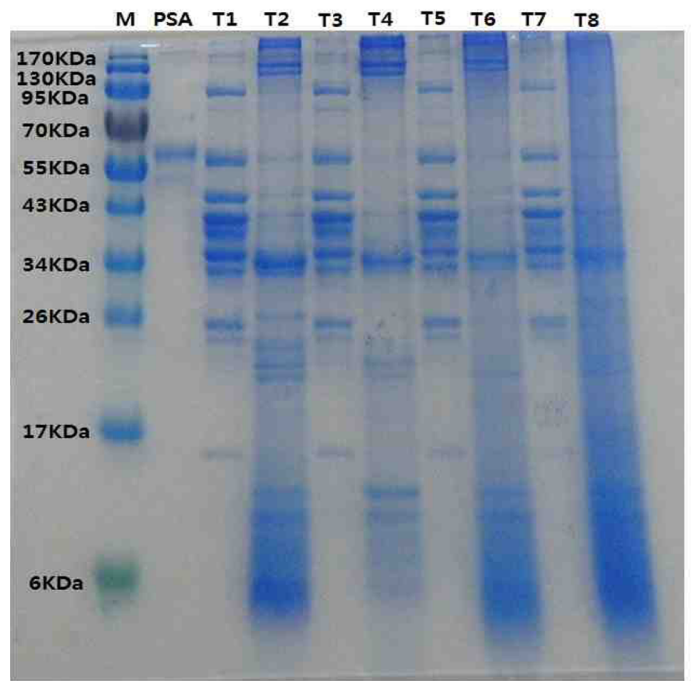

(B)

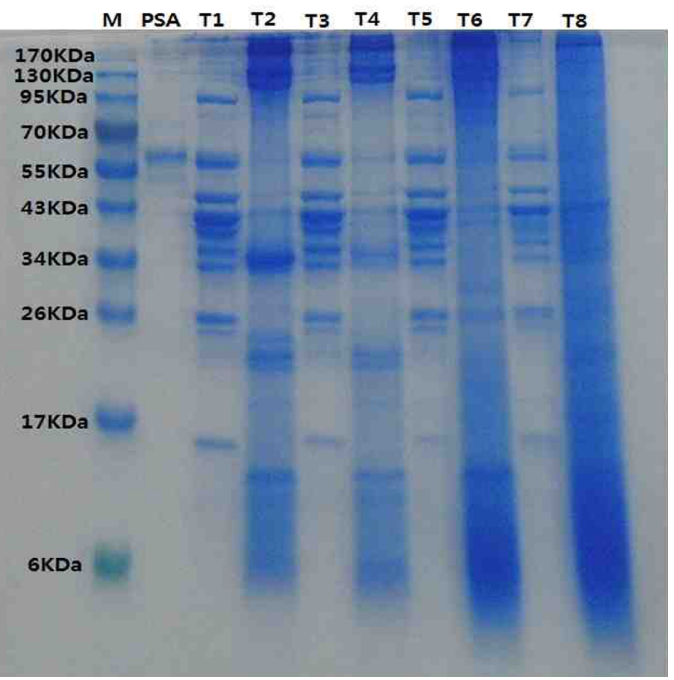

Fig. 2. SDS-PAGE profile of raw and broiled pork meat protein after marinating for 4 or $8 \mathrm{~h}$ with commercial sauce alone, commercial sauce with kiwi juice, and commercial sauce with pineapple juice. (A) SDS-PAGE profile of pork ham marinated for $4 \mathrm{~h}$ with or without subsequent broiling. (B) SDS-PAGE profile of pork ham marinated for $8 \mathrm{~h}$ with or without subsequent broiling. Lane M, molecular weight marker; lane PSA, commercial porcine serum albumin; lane T1, raw pork ham; lane T2, broiled pork ham; lane T3, raw pork ham marinated with commercial sauce alone; lane T4, pork ham marinated with commercial sauce and then broiled; lane T5, raw pork ham marinated with commercial sauce and kiwi juice; lane T6, pork ham marinated with commercial sauce and kiwi juice and then broiled; lane T7, raw pork ham marinated with commercial sauce and pineapple juice; lane T8, pork ham marinated with a commercial sauce and pineapple juice and then broiled.

pork ham (RP) was soaked for $4 \mathrm{~h}$ or $8 \mathrm{~h}$ in a marinade containing $27 \%$ commercial sauce only (RPM), commercial sauce with $10 \%$ kiwi juice (RPMK), or a commercial sauce with $10 \%$ pineapple juice (RPMP) (Table 1). Then, it was either analyzed immediately or subjected to pan broiling prior to analysis and designated as BP, BPM, BPMK, and BPMP. In addition, pork that was pan broiled without prior marination was examined.

\section{SDS-PAGE profiles of PSA after marination}

Fig. 2 shows the protein profiles of pork that was marinated for 4 or $8 \mathrm{~h}$ in commercial sauce only, commercial sauce and kiwi juice, or commercial sauce and pineapple juice and then either analyzed immediately or pan broiled prior to analysis. An intense band corresponding to the size expected for PSA (approximately $66 \mathrm{kDa}$ ) was observed in extracts from raw pork meat that had been marinated with commercial sauce alone, commercial sauce and kiwi juice, and commercial sauce and pineapple juice for both 4 and $8 \mathrm{~h}$. However, broiling pork meat at $300^{\circ} \mathrm{C}$ for $4 \mathrm{~min}$ after marination significantly decreased the intensity of the band, although it was not completely eliminated, and also significantly reduced the intensities of other bands at approximately $6 \mathrm{kDa}, 17 \mathrm{kDa}, 26 \mathrm{kDa}$, and $43 \mathrm{kDa}$. In addition, marinating for $8 \mathrm{~h}$ caused an increase in the intensity of a band that was less than $6 \mathrm{kDa}$ in size. Interestingly, it has been reported that the allergenicity of a protein may be either decreased or increased by food processing steps as epitope structures are destroyed or new epitopes are formed (Besler et al., 2001; Kim et al., 2011).

Kiwi and pineapple are the most popular fruits used to tenderize meat. Actinidin is a cysteine protease present in kiwi fruit, and it belongs to the same class of enzymes as ficin, papain, and bromelain. Actinidin hydrolyzes a wide range of myofibril proteins, including actomyosin (myosin and actin; 223 and $42 \mathrm{kDa}$, respectively), and Z-disk associated proteins such as nebulin and titin (650-3200 $\mathrm{kDa})$, filamin $(280 \mathrm{kDa})$, actinin $(100 \mathrm{kDa})$, and desmin $(53 \mathrm{kDa})$, although in a nonselective manner (Ha et al., 2012). When the tenderizing effects of actinidin and papain on bovine semitendinosus muscle were compared, a higher amount of actinidin was needed in order to produce tenderness equivalent to that observed using papain, although, unlike papain, actinidin did not overtenderize the meat. In addition, actinidine treatment caused myosin heavy chain proteins to be degraded and led to changes in the intensities of bands associated with other myofibrillar proteins (Ha et al., 2012). Moreover, treatment with actinidin did not produce off flavors or odors in the meat 
(A)

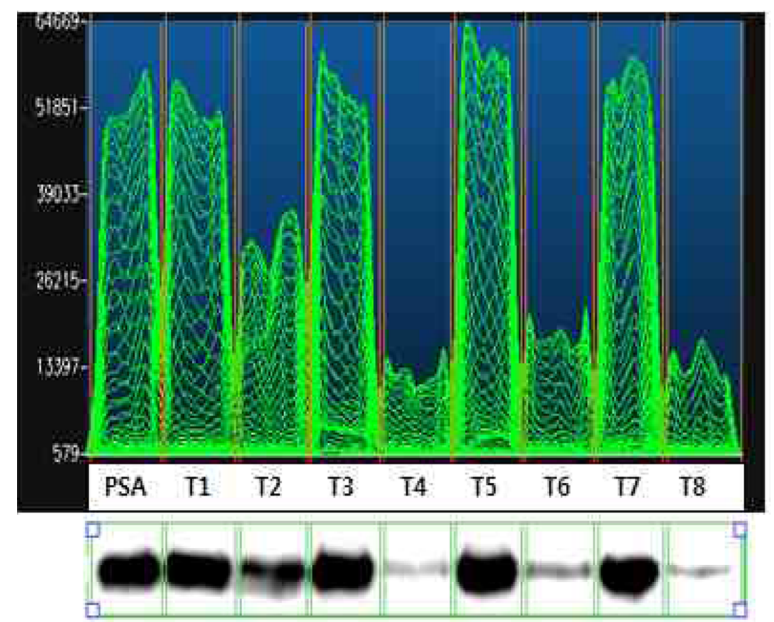

(B)

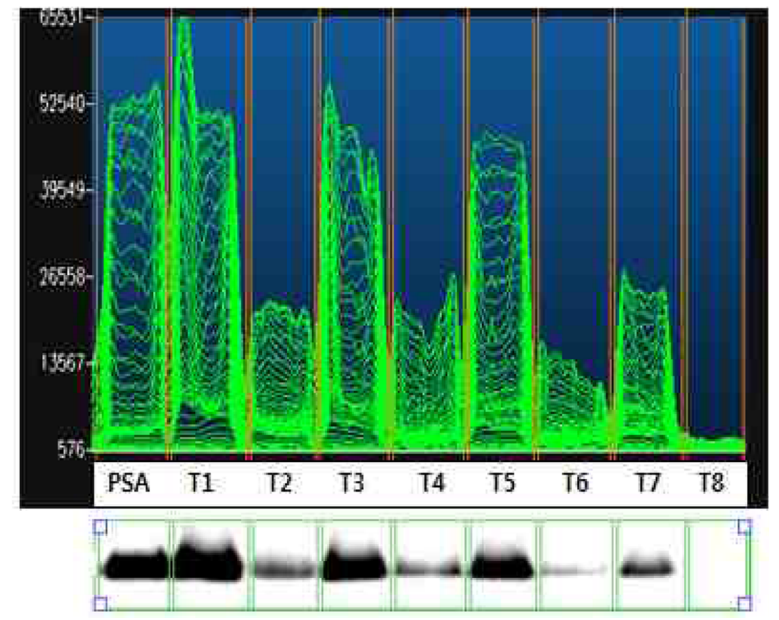

Fig. 3. Immunoblotting assay for PSA from raw and broiled pork meat protein after marinating for 4 or $8 \mathrm{~h}$ with commercial sauce alone, commercial sauce with kiwi juice, and commercial sauce with pineapple juice. (A) Immunoblotting profile of pork ham marinated for $4 \mathrm{~h}$ with or without subsequent broiling. (B) Immunoblotting profile of pork ham marinated for $8 \mathrm{~h}$ with or without subsequent broiling. Lane PSA, porcine serum albumin; lane T1, raw pork ham; lane T2, broiled pork ham; lane T3, raw pork ham marinated with commercial sauce alone; lane T4, pork ham marinated with commercial sauce alone and then broiled; lane T5, raw pork ham marinated with commercial sauce and kiwi juice; lane T6, pork ham marinated with commercial sauce and kiwi juice and then broiled; lane T7, raw pork ham marinated with commercial sauce and pineapple juice; lane T8, pork ham marinated with commercial sauce and pineapple juice and then broiled.

and did not cause surface "mushiness" (Kim and Taub, 1991). Bromelain (a cystein protease) is an enzyme mixture extracted from pineapple. Many studies reported that enzyme activity of bromelain is sustained without a marked decrease up to $80^{\circ} \mathrm{C}$ (El-Gharbawi and Whitaker, 1963; Tappel et al., 1956). Bromelain first degrades $40 \%$ of the collagen in the sarcolemma followed by degradation of myosin in the myofibrillar component (Kang and Rice, 1970; Wang et al., 1958).

\section{PSA expression in pork after marinating and broil- ing}

Levels of PSA in pork meat after marinating for $4 \mathrm{~h}$ with fruits extracts and subsequent pan broiling were determined by immunoblotting and are shown in Fig. 3. PSA was detected in both RP and RPM (Fig. 3(A)). However, the level of PSA was significantly reduced when marination and pan broiling were combined. Moreover, the length of marination time has an effect on the amount of PSA degraded, since the levels of PSA in samples after $8 \mathrm{~h}$ of marination (Fig. 3(B)) were less than those of samples marinated for $4 \mathrm{~h}$ (Fig. 3(A)). In addition, marination with pineapple juice, which contains bromelain, caused a more significant decrease in PSA than marination with kiwi juice. This may due to a difference in the activities of enzymes in kiwis and pineapples. Kim et al. (2010) su- ggested that the protease effect of crude extract from the pineapple on casein and myofibrillar protein was higher than that of extracts from kiwi fruits. In this study we focused on the change of porcine serum albumin amounts of pork meat by fruits juice with marination and heating and did not check enzyme activities in fruits juice. Further study is needed to evaluate exact change of enzyme activities during cooking and preparation. Sakai et al. (1998) reported that peptides less than 10,000 Da with very low IgE-binding activity could be produced after enzymatic treatment of pork gelatin (heat-denatured collagen) with collagenase. In this study, we found that heating and $8 \mathrm{~h}$ of marination with pineapple juice significantly decreased IgG binding activity. This result is well supported by the results from a study by Kim et al. (2011) on the effect of digestive enzymes such as pepsin and trypsin on the allergenicity of pork sausages. In that study, it was reported that pepsin and trypsin significantly reduced IgG binding activity up to $20 \%$.

To determine the exact concentration of PSA in the treated meat, sandwich ELISA assays were performed (Table 2 ), and the R squared value of the mean standard curve of PSA was found to be 0.995 . RP had a significantly higher level of PSA than broiled pork meat. The level of PSA in RPMP was significantly less than that RPM and RPMK. Interestingly, the levels of PSA after $8 \mathrm{~h}$ of marination in 
Table 2. Porcine serum albumin (PSA) content in pork meat as determined by sandwich ELISA (ng/mL)

\begin{tabular}{c|cccc|cccc}
\hline \hline \multirow{2}{*}{$\begin{array}{c}\text { Marinating time } \\
\text { (hrs) }\end{array}$} & \multicolumn{4}{|c|}{ Raw pork ham } & \multicolumn{4}{c}{ Broiled pork ham } \\
\cline { 2 - 8 } & RP & RPM & RPMK & RPMP & BP & BPM & BPMK & BPMP \\
\hline \multirow{2}{*}{4} & 5192.3 & 5559.1 & 5499.1 & 4711.8 & 111.5 & 38.2 & 16.3 & 8.6 \\
& $\pm 48.40^{\mathrm{Aa}}$ & $\pm 50.95^{\mathrm{Aa}}$ & $\pm 69.10^{\mathrm{Aa}}$ & $\pm 197.20^{\mathrm{Ba}}$ & $\pm 0.26^{\mathrm{Aa}}$ & $\pm 3.34^{\mathrm{Ba}}$ & $\pm 0.11^{\mathrm{Ba}}$ & $\pm 0.24^{\mathrm{Ca}}$ \\
\multirow{2}{*}{8} & 5060.4 & 3398.5 & 3002.9 & 1637.4 & 95.4 & 43.3 & 14.3 & 5.4 \\
& $\pm 108.65^{\mathrm{Aa}}$ & $\pm 172.40^{\mathrm{Bb}}$ & $\pm 18.20^{\mathrm{Cb}}$ & $\pm 108.85^{\mathrm{Db}}$ & $\pm 0.48^{\mathrm{Ab}}$ & $\pm 0.26^{\mathrm{Ba}}$ & $\pm 0.98^{\mathrm{Ca}}$ & $\pm 0.08^{\mathrm{Db}}$ \\
\hline
\end{tabular}

RP, raw pork ham; RPM, raw pork ham marinated with commercial sauce alone; RPMK, raw pork ham marinated with commercial sauce and kiwi juice; RPMP, raw pork ham marinated with commercial sauce and pineapple juice; $\mathrm{BP}$, pork ham broiled for 4 min at $300^{\circ} \mathrm{C}$; BPM, pork ham marinated with commercial sauce alone and then broiled; BPMK, pork ham marinated with commercial sauce and kiwi juice and then broiled; BPMP, pork ham marinated with commercial sauce and pineapple juice and then broiled.

both raw and broiled meat were significantly reduced. The PSA content of BP, BPM, BPMK, and BPMP was 95.4, 43.3, 14.3, and $5.4 \mathrm{ng} / \mathrm{mL}$, respectively $(p<0.05)$. These results shows that whereas marinating time and the use of pineapple juice in the marinade are important factors for reducing the level of PSA, cooking at $300^{\circ} \mathrm{C}$ has the most impact.

\section{Conclusions}

These results demonstrate that both cooking and marination with juices that contain cysteine proteases from the papain family such as actinidin from kiwi and bromelain from pineapple are effective for reducing levels of PSA in pork, and that the most effect method is to cook pork after marinating it in pineapple juice for $8 \mathrm{~h}$.

\section{Acknowledgements}

This work was carried out with the support of the Cooperative Research Program for Agriculture Science \& Technology Development (Project No. PJ009809) Rural Development Administration, Republic of Korea.

\section{References}

1. Besler, M., Steinhart, H., and Paschke, A. (2001) Stability of food allergens and allergenicity of processed foods. J. Chrom. $B$ 756, 207-228.

2. Chen, X., Feng, B. S., Zheng, P. Y., Liao, X. Q., Chong, J., Tang, S. G., and Yang, P. C. (2008) Fc gamma receptor signaling in mast cells links microbial stimulation to mucosal immune inflammation in the intestine. Am. J. Pathol. 173, 16471656.

3. Cho, E. D., Kim, D. S., and Jung, K. H. (2001) Identification of the chicken meat allergens. J. App. Pharm. 9, 7-14.

4. Chung, H. J., Park, J. H., Kim, J. H., Kim, Y. O., Chung, S.T., Kim, J. H., Cho, E. D., Cho, D. H., Noh, G. W., and Kim, D. S. (2001) Identification of allergens in pork meat. J. Pharm.
Korea 45, 39-45.

5. El-Gharbawi, M. and Witaker, J. R. (1963) Factors affecting enzymatic solubilization of beef proteins. J. Food Sci. 28, 168-172.

6. Fiocchi, A., Restani, P., and Riva, E. (2000) Beef allergy in children. Nutrition 16, 454-457.

7. Fisher, A. A. (1982) Allergic contact urticaria to raw beef: Hisopathology of the specific wheal reaction at the scratch test site. Contact Derm. 8, 425-425.

8. Ha, M., Bekhit, A. E. A., Carne, A., and Hopkins, D. L. (2012) Characterisation of commercial papain, bromelain, actinidin and zingibain protease preparations and their activities toward meat proteins. Food Chem. 134, 95-105.

9. Kang, C. K. and Rice, E. E. (1970) Degradation of various meat fractions by tenderizing enzymes. J. Food Sci. 35, 563565.

10. Kim, H. and Taub, I. A. (1991) Specific degradation of myosin in meat by bromelain. Food Chem. 40, 337-343.

11. Kim, K. B. W. R., Song, E. J., Lee, S. Y., Park, J. G., Lee, J. W., Byun, M. W., and Ahn, D. H. (2011) Changes in antigenicity of porcine serum albumin in gamma-irradiated sausage extract by treatment with pepsin and trypsin. Radiat. Phys. Chem. 80, 1258-1262.

12. Kim, M. H., Rho, J., and Kim, M. J. (2010) Proteolytic effect of fruit lesh and crude enzyme extract from fruits on myofibrilar protein. Korean J. Food Cookery Sci. 26, 323-329.

13. Korea Meat Trade Association (2013) Statistics of meat consumption. Available from: http://www.kmta.or.kr/eng/sub4-1. html?kej=eng\&scode. Accessed Jan. 23, 2014.

14. Laemmli, U. K. (1970) Cleavage of structural proteins during the assembly of the head of bacteriophage T4. Nature 227, 680-685.

15. Lee, S. I. (1993) The role of IgG in allergic disease. Pediatr. Allergy Respir. Dis. (Korea). 3, 3-10.

16. Sakai, Y., Yamato, R., Onuma, M., Kikuta, T., Watanabe, M., and Nakayama, T. (1998) Non-antigenic and low allergic gelatin produced by specific digestion with an enzyme-coupled matrix. Biol. Pharm. Bull. 21, 330-334.

17. Sicherer, S. H. and Sampson, H. A. (2010) Food allergy: Epidemiology, pathogenesis, diagnosis, and treatment. J. Allergy Clin. Immunol. 125, S116-S125.

18. Tappel, A. L., Miyada, D. S., Sterling, C., and Maier, V. P. 
(1956) Meat tenderization. II. Factors affecting the tenderization of beef by papain. Food Res. 21, 375-383.

19. Wang, C. H., Kou, S. K., and Chen, H. L. (2002) Porcine serum albumins sandwith ELISA for determining the cooking temperature of cured ground pork. Taiwanese J. Agric. Food Chem. 40, 197-204.
20. Wang, H., Weir, C. E., Birkner, M. L., and Ginger, B. (1958) Studies on enzymatic tenderization of meat. III. Histological and panel analyses of enzyme preparations from three distinct sources. Food Res. 23, 423-438.

(Received 2014.2.11/Revised 2014.5.17/Accepted 2014.5.20) 